\title{
Morfometria de Papilioninae (Lepidoptera, Papilionidae) ocorrentes em quatro localidades do Rio Grande do Sul, Brasil. I. Comparações com a massa corporal
}

\author{
Rocco Alfredo Di Mare ${ }^{1}$, Elio Corseuil ${ }^{2} \&$ Gustavo Schwartz $^{3}$
}

\begin{abstract}
'Departamento de Biologia, Centro de Ciências Naturais e Exatas (CCNE), Universidade Federal de Santa Maria (UFSM). Campus Universitário, Faixa de Camobi-km 9. 97 115-900. Santa Maria-RS, Brasil. Endereço eletrônico: ram13@ terra.com.br ${ }^{2}$ Instituto de Biociências, Pontifícia, Universidade Católica do Rio Grande do Sul (PUCRS), Curso de Pós-Graduação em Biociências. Caixa Postal 1429, 90619-900 Porto Alegre-RS, Brasil. Endereço eletrônico: corseuil@pucrs.br

${ }^{3}$ Departamento de Biologia, Universidade da Região da Campanha (URCAMP), Campus de São Gabriel. Rua Barão do Camobí 550, 97300-000 São Gabriel-RS, Brasil. Endereço eletrônico: schwartzgustavo@hotmail.com
\end{abstract}

\begin{abstract}
Morphometrics of Papilioninae (Lepidoptera, Papilionidae) occurring in four localities of Rio Grande do Sul, Brazil. I. Comparisons with body mass. Relationship analysis among wing and body parts in Lepidoptera may reveal a connection between structure and function. This study investigates relationships between swallowtail body size and its parts to understand how shape and size are determined, and how they are related to adaptation to biotic and abiotic environments. Females are heavier than males (up to $1,75 \mathrm{~g}$ ). In different habitats, thorax and abdomen in females are larger in $100 \%$ of the 11 analyzed species, total mass $92 \%$, and wings mass $75 \%$. Species whose females had greater mass also showed greater movement and dispersion in their home range. In habitats more disturbed by man or with a lower canopy, the increase in thorax size is significantly larger than that of wings, for both, males as females. In males, the abdomen increase is not significantly larger than that of the wings, in all places investigated. However, in females the abdomen increase is significant in all areas. Cluster analysis shows two large groups characterized by species with similar sizes and proportions. One inhabits environments with high humidity, and the other (more heterogeneous) occupies habitats appreciably altered by human action. The first shows two subdivisions distinguished by the display type those males present to the females.
\end{abstract}

KEYwORDs. Allometry; body size; butterfly; flight behavior; swallowtail butterflies.

Resumo. A análise da relação entre a asa e partes do corpo em Lepidoptera podem revelar uma conexão entre a estrutura e a função. Este estudo investigou relações entre o tamanho do corpo de papilionídeos e suas partes, importantes para entender como a forma e o tamanho são determinados, e como eles estão adaptados aos ambientes bióticos e abióticos. Fêmeas são mais pesadas que machos (acima de $1,75 \mathrm{~g}$ ). Em habitat diferentes, o tórax e o abdômen das fêmeas é maior em $100 \%$ das 11 espécies analisadas, a massa total $92 \%$, e a massa de asas $75 \%$. Espécies cujas fêmeas tiveram a maior massa também mostraram maior deslocamento e dispersão nas suas áreas de vida. Em habitats mais perturbados pelo homem ou com menor cobertura vegetal, o aumento no tamanho do tórax é significativamente maior que o das asas, tanto para os machos como para as fêmeas. Nos machos, o aumento de abdômen não é significativamente maior que o das asas, em todos os locais investigados. Porém, nas fêmeas o aumento do abdômen é significativo em todas os locais. A análise de agrupamento mostra dois grandes grupos caracterizados por espécies com tamanhos e proporções semelhantes. Um grupo com espécies que ocupam ambientes com umidade alta, e outro (mais heterogêneo) que ocupa habitats alterados pela ação do homem. O primeiro mostra duas subdivisões distinguidas pelo tipo de exibição que machos apresentam às fêmeas.

Palavras-Chave: Alometria; borboleta; comportamento de vôo; papilionídeos; tamanho do corpo.

A evolução pode ser entendida como um processo determinado por duas hierarquias sobrepostas, a ecológica e a genealógica, através das quais os organismos são modelados (ELDREDGE 1986; FunK \& BROOKS 1990). Deste modo, o tamanho do corpo pode ser considerado um caráter evolutivo chave, rigorosamente relacionado com a morfologia, fisiologia, ecologia e história de vida (Peters 1983; CALDER 1984; BARBaULT 1988; La Barberra 1989; Reiss 1989; Fairbairn 1992). Geralmente, o tamanho corporal ótimo é atribuído a dois fatores: o primeiro fundamentado no balanço entre a reprodução máxima imediata e investimento energético no crescimento para aumentar a sobrevivência e sucesso reprodutivo (GADGIL \& Bossert 1970; Stearns \& Crandall 1984) e o segundo, em quanto do tamanho corporal é influenciado pela quantidade de energia que o organismo pode retirar do seu ambiente (Schoener 1969). As alterações no valor adaptativo relativo podem resultar de um conjunto de pressões ecológicas como: eficiência energética, termorregulação, predação, parasitismo, competição intra- e interespecífica, as quais atuam sobre o valor seletivo, usando o peso do corpo como uma variável central (BARBAULT 1988).

No processo evolutivo, alterações no tamanho do corpo são geralmente acompanhadas por alterações na forma. Forma e função são as bases da escala biológica em estudos de relações alométricas e de covariação do tamanho (SCHMIDT-NieLSEN 1984). A maior parte dos modelos evolutivos presume que as proporções alométricas procedem de seleção que atua no tamanho corporal como um todo (Gould 1966; BARBAult 1988; 
REEVE \& FAIRBAIRN 1996). Entretanto, alterações na forma podem também refletir seleção diferencial atuando em determinadas partes do corpo (LANDE 1979; Zeng 1988; RISKA 1989). Portanto, alterações na forma durante a ontogenia dependem de alterações no crescimento relativo de várias partes do corpo. No entanto, apesar das pressuposições dos modelos teóricos, o consenso corrente sobre o desenvolvimento é que os tamanhos finais de apêndices e órgãos internos são regulados autonomamente (NIJHOUT \& EMLEN 1998).

No contexto da alometria, o dimorfismo sexual é um caso interessante, pois machos e fêmeas de uma mesma espécie podem apresentar tamanhos e formas diferentes. Dentro de uma família, ou mesmo de um gênero, são comuns desvios que favorecem um dos sexos (FAIRBAIRN 1990; ARAK 1994). DARWIN (1871) foi um dos primeiros a salientar que entre invertebrados as fêmeas são geralmente maiores que os machos, ao contrário do que normalmente acontece no padrão dos vertebrados (Ghiselin 1974; Hendrick \& Temeles 1989; Hanken \& WaKe 1993; ANDERSEN 1994).

Certas partes do corpo podem ser influenciadas pelo ambiente (VITT \& CONGDON 1978). Diferenças no tamanho das partes do corpo podem estar associadas a diferenças no nicho ecológico dos organismos. Tendências em que duas espécies que ocupam posição similar num nicho ecológico, numa certa dimensão, divergem com relação à outra dimensão desse nicho, quando duas espécies, que coexistem no mesmo local, podem se diferenciar pela dieta alimentar. $\mathrm{O}$ resultado desta procura ou tendência em diferenciar-se, quanto ao uso dos recursos alimentares, pode ser exemplificado em lagartos e peixes cuja distribuição da massa é diferente, dependendo do modo de alimentação (VitT \& Congdon 1978; Huey \& Pianka 1981; WebB 1984). Contudo, esta desigualdade também pode ser resultado de diferenças na suscetibilidade à predação (HuEY \& PIANKA 1981; ViтT 1983). Quanto a borboletas, algumas podem apresentar uma distribuição de massa entre o tórax (massa muscular dedicada ao vôo) e o abdômen (massa reprodutiva e órgãos de digestão) variando de acordo com a palatabilidade das espécies a vertebrados predadores e que se reflete nas características do vôo (Chai \& SRygley 1990; SRygley \& Chai 1990; SRygley \& Dudley 1993; Dudley 2000).

Esse trabalho teve como objetivos estudar em Papilionidae: a) o relacionamento do tamanho total do corpo, bem como as proporções das partes como tórax, abdômen e asas, entre espécies e entre sexos, b) as proporções corporais entre borboletas capturadas em diferentes locais de amostragem, com diferenças na percentagem da cobertura vegetal e c) o relacionamento entre características comportamentais dos machos (nível de vôo, batidas das asas, estilo de vôo e de corte e deslocamentos nas áreas de vida) com as proporções das partes do corpo. De modo geral, o estudo estabelece relações entre o tamanho do corpo, e entre as suas partes, que podem auxiliar no entendimento de como a forma e o tamanho dos organismos são determinados, o papel que desempenham na adaptação das borboletas aos seus ambientes bióticos e abióticos e sua relação com o comportamento de vôo.

A escolha da família Papilionidae deveu-se ao fato de que as espécies, em conjunto, apresentam características que as tornam excelentes organismos para estudos alométricos e de correlação entre a massa corporal e os ambientes ocupados.

Papilionidae são borboletas com vôo ágil e poderoso, mas nem sempre rápido. São abundantes em ambientes úmidos, com grande abundância de árvores nativas e diversidade de microhábitats. Os machos apresentam androcônias com cheiro bem forte e característico, usados para apaziguar as fêmeas durante os cortejamentos aéreos vigorosos e prolongados. As fêmeas de muitas espécies são raramente vistas, até desconhecidas, tendo hábitos que evitam encontros com coletores, como voar no interior de matas densas, ou pendurarse como uma pipa a $50 \mathrm{~m}$ acima do chão, à procura de brotos novos em cima de plantas hospedeiras, árvores grandes da floresta. Esta é a família mais primitiva das borboletas "verdadeiras" (não incluindo os Hesperioidea, uma superfamília a parte), e inclui muitas espécies antigas, relituais, de distribuição muito restrita, à beira da extinção devido à destruição, pelo homem, dos seus poucos hábitats remanescentes (Brown Jr. 1992).

A família Papilionidae, apesar de cosmopolita, concentrase nos trópicos (SCRIBER et al. 1991), sendo formada por cerca de 560 espécies. Dentre estas, 143 são registradas para o continente americano, distribuídas em três subfamílias (FEENY 1991; TyLER et al. 1994). Para o Rio Grande do Sul, são referidas 37 espécies e subespécies (Teston \& Corseuil 1998). Os papilionídeos apresentam longevidade pequena e são especialmente vulneráveis a mudanças rápidas em seus ambientes, com algumas espécies bem adaptadas a ambientes antrópicos. Por exemplo, Heraclides anchisiades capys, ao se adaptar ao gênero Citrus (Rutaceae) (Tyler et al. 1994) e Pterourus scamander que se alimenta de Persea sp. (Lauraceae), bastante comum em áreas urbanas.

\section{MATERIAL E MÉTODOS}

Organismos estudados. O estudo foi fundamentado em onze espécies de Papilioninae, uma das quais com três subespécies, pertencentes a três tribos (Fig. 1). Graphiini (Mimoides lysithous lysithous (Hübner, 1821); Mimoides lysithous eupatorion (Lucas, 1857); Mimoides lysithous rurik (Eschscholtz, 1821). Papilionini (Heraclides astyalus astyalus (Godart, 1819); Heraclides hectorides (Esper, 1794); Heraclides thoas brasiliensis (Rothschild \& Jordan, 1906); Pterourus scamander scamander (Boisduval, 1836)). Troidini (Battus polydamas polydamas (Linné, 1758); Battus polystictus polystictus (Butler, 1874); Euryades corethrus (Boisduval, 1836); Parides agavus (Drury, 1782); Parides anchises nephalion (Godart, 1819); Parides bunichus perrhebus (Boisduval, 1836)).

Locais estudados. Os locais estudados situam-se entre os meridianos $53^{\circ} 10^{\prime}$ e $54^{\circ} 40^{\prime} \mathrm{W}$ e entre $29^{\circ} 00^{\prime}$ e $29^{\circ} 20^{\prime} \mathrm{S}$. As localidades de Água Negra (AN), Morro do Link (ML), Rincão do Canto (RC), Rincão do Soturno (RS), e Vale do Diabo (VD), estão em áreas remanescentes da floresta decidual e semidecidual da Serra Geral, com altitude inferior a $500 \mathrm{~m}$. Neles 
podem ser observados cinco extratos vegetais: emergente, arbóreo contínuo, arvoretas, arbustivo e herbáceo, com cerca de 200 espécies arbóreas formam a mata local, sendo que 135 são exclusivas (KLEIN 1984). Schöenstatt (SC) e Camobi (CB) são áreas eminentemente urbanas, com flora caracterizada por plantas ornamentais cultivadas, tais como: Apocynaceae, Asteraceae, Malvaceae, Myrtaceae e Verbenaceae. Schöenstatt apresenta maior número de espécies do que Camobi.

As áreas investigadas foram agrupadas segundo SCHWARTZ $\&$ Di MARE (2001), de acordo com a cobertura vegetal presente, identificando dois grandes grupos: o primeiro é formado por áreas localizadas em zonas urbanas, denominado SCCB (SC+ $\mathrm{CB}$ ), e o segundo é constituído por dois subgrupos: um inclui áreas de floresta, com cobertura vegetal entre $50 \%(\mathrm{RSML}=\mathrm{RS}$ $+\mathrm{ML})$ e $70 \%(\mathrm{VDRC}=\mathrm{VD}+\mathrm{RC})$ e o outro formado por $\mathrm{AN}$, cuja cobertura é de cerca de 30\%. Água Negra se distinguiu das demais áreas por suas características fisionômicas particulares: hábitat fragmentado com vegetação lenhosa baixa e de média altura, observada nos capões, com abundância de Myrtaceae e Rubiaceae.

No trabalho, os agrupamentos passam a ser referidos como comunidades, por conveniência e por afinidade com a cobertura vegetal.

Em AN, RC, RS e VD as coletas foram realizadas em trilhas e estradas existentes, previamente escolhidas. Em CB foram percorridas ruas do bairro, escolhidas pelo número de casas com jardins apresentando flores. Em SC foi investigada uma pequena mata secundária, com cerca de $5000 \mathrm{~m}^{2}$.

Amostragem e identificação. As coletas ocorreram de setembro de 1994 a março de 1995 e de novembro de 1995 a fevereiro de 1996. Foram realizadas em intervalos médios de trinta dias para cada localidade estudada, utilizando duas redes entomológicas convencionais, durante intervalos médios de quatro horas. As capturas eram iniciadas entre nove e dez horas da manhã. As capturas eram iniciadas entre nove e dez horas da manhã. Os imagos capturados foram acondicionados em envelopes de papel vegetal, individualizados, devidamente etiquetados e transportados para o Laboratório de Biologia Evolutiva do Departamento de Biologia da UFSM. Em laboratório foram executados três tipos de pesagens: imagos inteiros, sem as asas e somente o abdômen. A massa torácica (incluindo cabeça e patas) foi estimada pela subtração do abdômen da massa total sem as asas. A separação das partes foi realizada com uma tesoura cirúrgica de ponta fina e as pesagens realizadas com uma balança de precisão até $0,1 \mathrm{mg}$. Salienta-se que todas as pesagens foram realizadas logo após a captura no campo, com intervalo de aproximadamente quatro horas entre a captura e a pesagem. As espécies foram identificadas a partir de TYLER et al. (1994). Em algumas análises não foram utilizadas todas as borboletas capturadas, por exemplo, as que incluem as asas. Foram excluídas aquelas cujas asas estavam danificadas.

Parâmetros estudados e análise de dados. As comparações foram efetuadas com base nas medidas da massa do corpo, das

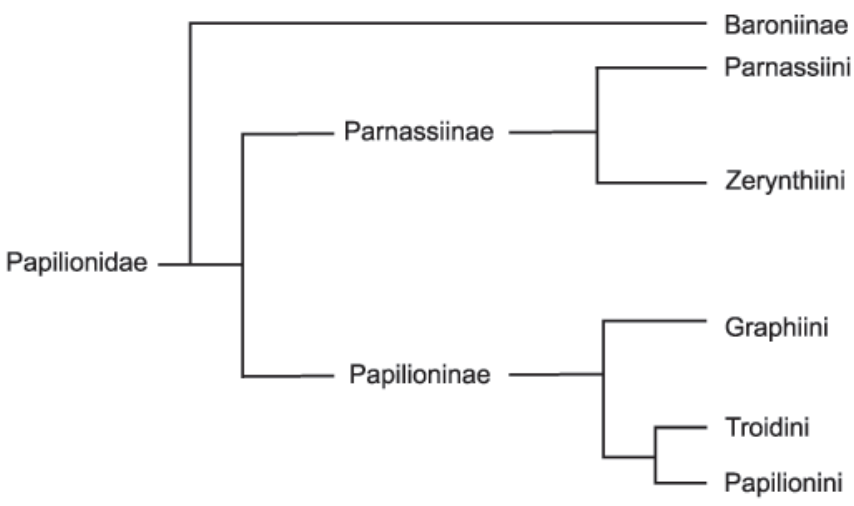

Fig. 1. Filogenia das tribos de Papilionidae (de acordo com Miller 1987)

asas, do tórax e do abdômen. Na análise estatística as variáveis foram transformadas (transformação logarítmica) quando não satisfaziam os critérios de normalidade e homocedasticidade.

Deste modo, foram estimados os coeficientes de correlação para as espécies, individualmente ou agrupadas, cujos sexos foram analisados separadamente. Os valores discordantes entre as correlações estimadas, para ambos os sexos, foram comparadas através de teste bicaudal, que analisa se as diferenças são significativas.

As médias foram confrontadas através dos testes de comparações múltiplas "Least Square Significant" (LSD) e Bonferroni. Estes métodos de comparação levam em conta, especificamente, a utilização de mais de duas amostras. O primeiro baseia-se no número de exemplares dos grupos envolvidos na comparação. Ele oferece proteção mínima em relação a um aumento na taxa de erro alfa, devido às comparações múltiplas a posteriori (ZAR 1999). O segundo ajusta o nível alfa de cada teste individual para assegurar que o risco global para vários testes permaneça 0,05 .

Análise de agrupamentos. Em estudos ecológicos é tendência normal procurar agrupar ou associar as espécies nas comunidades. Tal procedimento visa descrever a estrutura de um ecossistema determinando a composição e a extensão das suas unidades funcionais, reconhecendo entre elas um grau de similaridade suficiente para reuni-las em um mesmo conjunto (VALENTIN 2000). Este confronto foi realizado através da análise de agrupamento, a partir das médias estimadas para as massas do tórax e do abdômen dos machos de cada espécie investigada, nas diferentes localidades. A investigação do comportamento foi realizada somente com os machos porque as fêmeas eram geralmente menos abundantes e o número de exemplares que puderam ser utilizados nas análises foi bem menor. Os agrupamentos formados desta maneira foram confrontados com algumas características comportamentais dos machos, como: nível de vôo, batida das asas e estilo de vôo, estilo de corte, movimentos individuais ou de migração e ambiente utilizado. Os comportamentos utilizados para formar os grupos funcionais e ecológicos foram estudados a partir de observações de campo, tendo como base critérios inferidos em BROwN Jr. (1992) e TYLER 


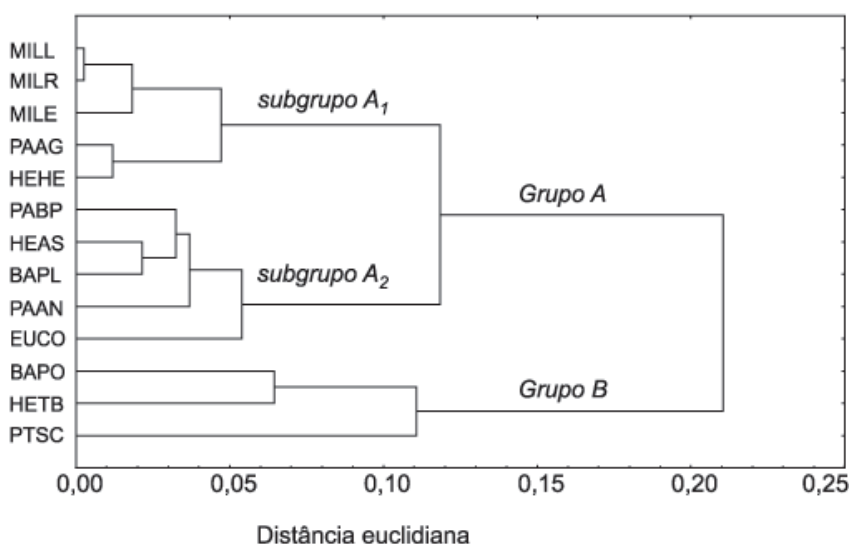

Fig 2. Agrupamento das espécies estudadas a partir das médias e desvios padrões das massas torácica e abdominal $(\mathrm{MILL}=$ M. lysithous lysithous; $\mathrm{MILR}=M$. lysithous rurik; $\mathrm{MILE}=M$. lysithous eupatorion; $\mathrm{PAAG}=P$. agavus; $\mathrm{HEHE}=H$. hectorides; $\mathrm{PABP}=P$. bunichus perrhebus; $\mathrm{PAAN}=$ $P$. anchises nephalion; $\mathrm{HEAS}=H$. astyalus astyalus; $\mathrm{BAPL}=B$. polydamas polydamas; $\mathrm{EUCO}=$ E. corethrus $; \mathrm{BAPO}=B$. polystictus polystictus; $\mathrm{HETB}=H$. thoas brasiliensis; $\mathrm{PTSC}=P$. scamander scamander $)$, utilizando o método UPGMA e distância euclidiana.

et al. (1994). Os agrupamentos foram formados através do mé0todo UPGMA (SNEATH \& SOKAL 1973), utilizando a distância euclidiana como parâmetro de comparação. Este método calcula a média aritmética da similaridade (ou da distância) entre o objeto que se quer incluir num grupo e cada objeto desse grupo. O objeto é atribuído ao grupo com o qual ele tem a maior similaridade média (ou menor distância média) considerando todos os objetos (VALENTIN 2000).

\section{RESULTADOS EDISCUSSÃO}

Comparações entre as partes do corpo. Com base em 704 exemplares (499 machos e 205 fêmeas), os resultados mostram que as proporções entre as partes estudadas são estatisticamente significantes. A relação entre o tórax e asas apresentou o maior grau de correlação, em ambos os sexos. Nas fêmeas o abdômen está mais relacionado com o tórax e nos machos com as asas.

$\mathrm{Na}$ Tabela I são apresentadas as correlações entre a massa das asas e a do tórax (A), entre a massa das asas e a do abdômen (B) e entre a massa do tórax e a do abdômen $(C)$, por espécie e por sexo. As espécies quando analisadas em conjunto, para as asas e o tórax (A), mostraram índices de correlação elevados e significativos, tanto para os machos $\left(\mathrm{r}_{\text {(asa x tórax) }}=0,88 ; \mathrm{n}=373 ; \mathrm{p}\right.$ $<0,01)$ como para as fêmeas $\left(\mathrm{r}_{\text {(asa x tórax) }}=0,91 ; \mathrm{n}=149 ; \mathrm{p}<0,01\right)$. A diferença entre os coeficientes de correlação dos machos e o das fêmeas não foi significante $(p>0,05)$, quando confrontados através de um teste bicaudal.

Duas espécies entre os machos (M. lysithous lysithous, e $P$. scamander scamander) e apenas uma entre as fêmeas $(P$. scamander scamander) não apresentaram correlações significativas. As correlações fenotípicas estimadas para as espécies individualmente, provavelmente, expressem também padrões subjacentes de correlação genética.

O coeficiente de correlação entre a massa das asas e a do abdômen (B), obtido com as espécies agrupadas, também foi alto e significante, para ambos os sexos (machos: $\mathrm{r}_{\text {(asas x abdômen) }}=$ 0,$78 ; \mathrm{n}=373 ; \mathrm{p}<0,01$ e fêmeas: $\mathrm{r}_{\text {(tórax x abdômen) }}=0,81 ; \mathrm{n}=149 ; \mathrm{p}<$ $0,01)$. A diferença nos valores médios entre os sexos, também, não foi significativa ( $\mathrm{p}>0,05)$. Individualmente, duas espécies nos machos (E. corethrus e P. scamander scamander) e seis espécies nas fêmeas, com uma subdividida em três subespécies (B. polystictus polystictus, $H$. astyalus astyalus, $H$. hectorides, $H$. thoas brasiliensis, M. lysithous lysithous, M. lysithous eupatorion, $M$. lysithous rurik, e P. scamander scamander) não expressaram correlações significativas.

A ausência de significância nas fêmeas entre a massa das asas e a do abdômen, em mais da metade das espécies investigadas, pode ser atribuída ao baixo número de exemplares examinados em cada espécie. Nos machos, com um número maior de borboletas analisadas por espécie, apenas as três espécies com baixo número de indivíduos não mostraram significância. Outra explicação para a ausência de significância poderia ser atribuída a fatores, que influenciariam o tamanho abdominal, não identificados neste trabalho.

As massas do tórax e do abdômen $(\mathrm{C})$ igualmente exibiram correlações significativas, tanto para os machos ( $\mathrm{r}_{\text {(tórax x abdômen) }}=$ $0,71 ; \mathrm{n}=499 ; \mathrm{p}<0,01)$ como para as fêmeas $\left(\mathrm{r}_{\text {(tórax x abdômen) }}=0,87\right.$; $n=205 ; \mathrm{p}<0,01)$, bem como nas outras comparações quando as espécies são agrupadas. As diferenças nos valores médios entre ambos os sexos foram significativos $(p<0,01)$. Embora significantes para a maioria das espécies, algumas correlações individuais dos machos (E. corethrus, M. lysithous lysithous, $M$. lysithous rurik e $P$. scamander scamander) e das fêmeas (M. lysithous lysithous e $M$. lysithous rurik) não o foram. Aqui também, a falta de correlação observada pode ser atribuída ao número de indivíduos analisados em cada espécie ou à independência entre as características analisadas nos machos.

Relação da massa corporal com sexo. As fêmeas, em média, foram mais pesadas que os machos (Tabela II), com taxas (massa média das fêmeas/ machos) que variam de 1,10 (E. corethrus) até 1,75 ( $B$. polystictus polystictus). A comparação dos coeficientes de variação, entre os sexos, levando-se em conta todos os locais de captura, mostrou que as fêmeas apresentaram valores mais elevados para a massa do tórax em 100\% das espécies estudadas, para a massa do abdômen e total em $90 \%$ e para a massa das asas em 33\%. Valores médios mais baixos para as quatro variáveis estimadas foram verificados em ambos os sexos, nas três subespécies de Mimoides lysithous. Os mais elevados foram obtidos com $H$. thoas brasiliensis e $P$. scamander scamander, também em ambos os sexos. Em $B$. polystictus polystictus somente as fêmeas se distinguiram com valores mais altos.

Após uma revisão realizada nos invertebrados, ReISs (1989) concluiu que as fêmeas superam o tamanho dos machos somente quando o investimento energético para a reprodução for pequeno. Tal estratégia pode ser reivindicada para as fêmeas de papilionídeos. Entretanto, o mesmo não pode ser atribuído 
Tabela I. Correlação (r) entre a massa da asa e o tórax (A), asa e abdômen (B) e tórax e abdômen (C) dos papilionídeos capturados (n), nas localidades investigadas.

\begin{tabular}{|c|c|c|c|c|c|c|c|c|c|c|c|c|c|c|c|c|c|c|}
\hline & \multicolumn{6}{|c|}{ A } & \multicolumn{6}{|c|}{ B } & \multicolumn{6}{|c|}{$\mathrm{C}$} \\
\hline & \multicolumn{6}{|c|}{$\begin{array}{l}\text { Massa das asas } \\
\text { x } \\
\text { Massa torácica }\end{array}$} & \multicolumn{6}{|c|}{$\begin{array}{c}\text { Massa das asas } \\
\mathrm{x} \\
\text { Massa do abdômen }\end{array}$} & \multicolumn{6}{|c|}{$\begin{array}{c}\text { Massa torácica } \\
\text { x } \\
\text { Massa do abdômen }\end{array}$} \\
\hline & \multicolumn{3}{|c|}{ Machos } & \multicolumn{3}{|c|}{ Fêmeas } & \multicolumn{3}{|c|}{ Machos } & \multicolumn{3}{|c|}{ Fêmeas } & \multicolumn{3}{|c|}{ Machos } & \multicolumn{3}{|c|}{ Fêmeas } \\
\hline & $\mathrm{r}$ & $\mathrm{n}$ & $\mathrm{p}$ & $\mathrm{r}$ & $\mathrm{n}$ & $\mathrm{p}$ & $\mathrm{r}$ & $\mathrm{n}$ & $\mathrm{p}$ & $\mathrm{r}$ & $\mathrm{n}$ & $\mathrm{p}$ & $r$ & $\mathrm{n}$ & $\mathrm{p}$ & $\mathrm{r}$ & $\mathrm{n}$ & $\mathrm{p}$ \\
\hline MILE & 0,48 & 15 & $*$ & 0,96 & 5 & $* *$ & 0,83 & 15 & $* *$ & 0,78 & 5 & $\mathrm{NS}$ & 0,48 & 19 & $*$ & 0,86 & 6 & $*$ \\
\hline MILL & 0,37 & 8 & NS & 0,98 & 4 & $*$ & 0,78 & 8 & $*$ & 0,18 & 4 & NS & 0,05 & 8 & NS & 0,12 & 5 & NS \\
\hline MILR & 0,84 & 5 & $* *$ & 0,75 & 9 & $*$ & 0,69 & 8 & $*$ & 0,46 & 9 & NS & 0,42 & 10 & NS & 0,48 & 10 & NS \\
\hline BAPL & 0,85 & 32 & $* *$ & 0,89 & 12 & $* *$ & 0,74 & 32 & $* *$ & 0,84 & 2 & $* *$ & 0,74 & 47 & $* *$ & 0,76 & 15 & $* *$ \\
\hline BAPO & 0,85 & 50 & $* *$ & 0,55 & 10 & $*$ & 0,57 & 50 & $* *$ & 0,34 & 10 & NS & 0,64 & 59 & $* *$ & 0,78 & 15 & $* *$ \\
\hline EUCO & 0,84 & 5 & $* *$ & 0,82 & 9 & $* *$ & 0,53 & 5 & NS & 0,67 & 9 & $*$ & - & 5 & $\mathrm{NS}$ & 0,75 & 10 & $*$ \\
\hline PAAG & 0,63 & 101 & $*$ & 0,91 & 21 & $* *$ & 0,58 & 101 & $* *$ & 0,82 & 21 & $* *$ & 0,71 & 136 & $* *$ & 0,85 & 34 & $* *$ \\
\hline PAAN & 0,82 & 67 & $* *$ & 0,83 & 47 & $* *$ & 0,83 & 67 & $* *$ & 0,72 & 47 & $* *$ & 0,81 & 84 & $* *$ & 0,80 & 58 & $* *$ \\
\hline PABP & 0,86 & 36 & $* *$ & 0,86 & 12 & $* *$ & 0,75 & 36 & $* *$ & 0,90 & 12 & $* *$ & 0,72 & 52 & $* *$ & 0,83 & 16 & $* *$ \\
\hline HEAS & 0,77 & 20 & $* *$ & 0,92 & 5 & $*$ & 0,73 & 20 & $* *$ & 0,45 & 5 & $\mathrm{NS}$ & 0,65 & 30 & $* *$ & 0,78 & 9 & $*$ \\
\hline HEHE & 0,53 & 15 & $*$ & 0,84 & 7 & $*$ & 0,66 & 15 & $* *$ & 0,69 & 7 & NS & 0,45 & 23 & $* *$ & 0,83 & 10 & $* *$ \\
\hline HETB & 0,97 & 8 & $* *$ & 0,98 & 4 & $*$ & 0,80 & 8 & $*$ & 0,55 & 4 & NS & 0,83 & 13 & $* *$ & 0,91 & 5 & $*$ \\
\hline PTSC & 0,31 & 8 & $\mathrm{NS}$ & 0,19 & 4 & $\mathrm{NS}$ & 0,16 & 8 & $\mathrm{NS}$ & 0,03 & 4 & $\mathrm{NS}$ & 0,51 & 13 & NS & 0,79 & 11 & $* *$ \\
\hline TOTAL & 0,88 & 373 & $* *$ & 0,91 & 149 & $* *$ & 0,78 & 373 & $* *$ & 0,81 & 149 & $* *$ & 0,71 & 499 & $* *$ & 0,87 & 205 & $* *$ \\
\hline
\end{tabular}

$\mathrm{MILE}=M$. lysithous eupatorion $; \mathrm{MILL}=$ M. lysithous lysithous; $\mathrm{MILR}=$ M. lysithous rurik $; \mathrm{BAPL}=B$. polydamas polydamas; $\mathrm{BAPO}=$ B. polystictus polystictus $; \mathrm{EUCO}=$ E. corethrus $; \mathrm{PAAG}=P$. agavus $; \mathrm{PAAN}=P$. anchises nephalion $; \mathrm{PABP}=P$. bunichus perrhebus $; \mathrm{HEAS}=H$. astyalus astyalus $;$ $\mathrm{HEHE}=H$. hectorides $\mathrm{HETB}=H$. thoas brasiliensis; $\mathrm{PTSC}=P$. scamander scamander.

$\mathrm{p}=$ significância da correlação; $*=\mathrm{p}<0,05 ; * *=\mathrm{p}<0,01$; ns= não significante

aos machos, pois o investimento é muito grande pelo domínio de espaços, fêmeas e/ou locais para acasalamento (LEDERHOUSE 1982). Além do mais, pode-se invocar que as diferenças observadas entre os sexos podem ser atribuídas a forças seletivas diferentes, por outras razões não associadas ao acasalamento, tal como, por diferenciação ecológica, onde os dois sexos estão adaptados a nichos ecológicos diferentes. Esta idéia foi inicialmente sugerida por WALLACE (apud CRONIN 1995). Machos e fêmeas explorariam recursos alimentares diferentes, porque ambos se beneficiariam da competição reduzida por um suprimento limitado (SELANDER1972).

Embora tal comportamento não tenha sido registrado em Papilionidae, segundo CRONIN (1995) as fêmeas de algumas espécies podem fazer um julgamento não específico, selecionando "capacidades" ou "qualidades", como por exemplo: velocidade, força e/ou agilidade (CRONIN 1995).

As espécies com elevadas taxas entre a massa média das fêmeas/machos, como ocorre em B. polystictus polystictus $(1,76)$, P. scamander scamander $(1,66)$, P. bunichus perrhebus $(1,62), H$. thoas brasiliensis $(1,56), H$. hectorides $(1,54)$ e $H$. astyalus $(1,27)$, podem ser associadas a uma maior movimentação e/ou dispersão dentro da área de vida.

Relação da massa corporal com os locais de captura. A Tabela III (A) mostra que os valores médios mais elevados para a massa total foram obtidos em SCCB. A diferença entre a média de SCCB e as demais foi estatisticamente significante, quando testada através dos testes LSD e Bonferroni, tanto para os machos como para as fêmeas. Já, as diferenças entre as médias estimadas para VDRC, RSML e AN, com o teste LSD, foram significativas apenas para os machos. Com o teste de Bonferroni, tanto para os machos como para as fêmeas, não foram significantes. Contudo, quando os valores médios são analisados individualmente para os machos de cada espécie, os mais elevados, em $100 \%$ das espécies, podem ser observados em AN, RSML e VDRC e não em SCCB (Tabela II). Nas fêmeas ocorreu em $57 \%$ das espécies. Na literatura, existem dados de animais em competição por locais ou fêmeas mostrando que os vencedores normalmente apresentam maior tamanho (ARCHER 1988), ou maior massa muscular que seus adversários (CONVEY 1989). Desse modo, a dualidade aparente, entre a média estimada para as localidades e espécies, poderia ser atribuída ao fato que as borboletas de florestas, onde as condições são mais propícias, as fontes alimentares são mais abundantes e/ou mais nutritivas, apresentariam tamanhos maiores, quando comparadas com as de áreas mais marginais. Por outro lado, em áreas marginais cujas condições não são ótimas, indivíduos ou espécies com tamanhos maiores teriam mais chances de sobreviver e/ou se reproduzirem nestes locais (DYCK \& MatTHYSEN 1999).

Esta relação é bastante razoável quando comparamos a proporção tórax/abdômen (Tabela III B) das espécies agrupadas por local. Conforme a cobertura vegetal das áreas vai decrescendo e a ação antrópica aumentando, os valores médios 


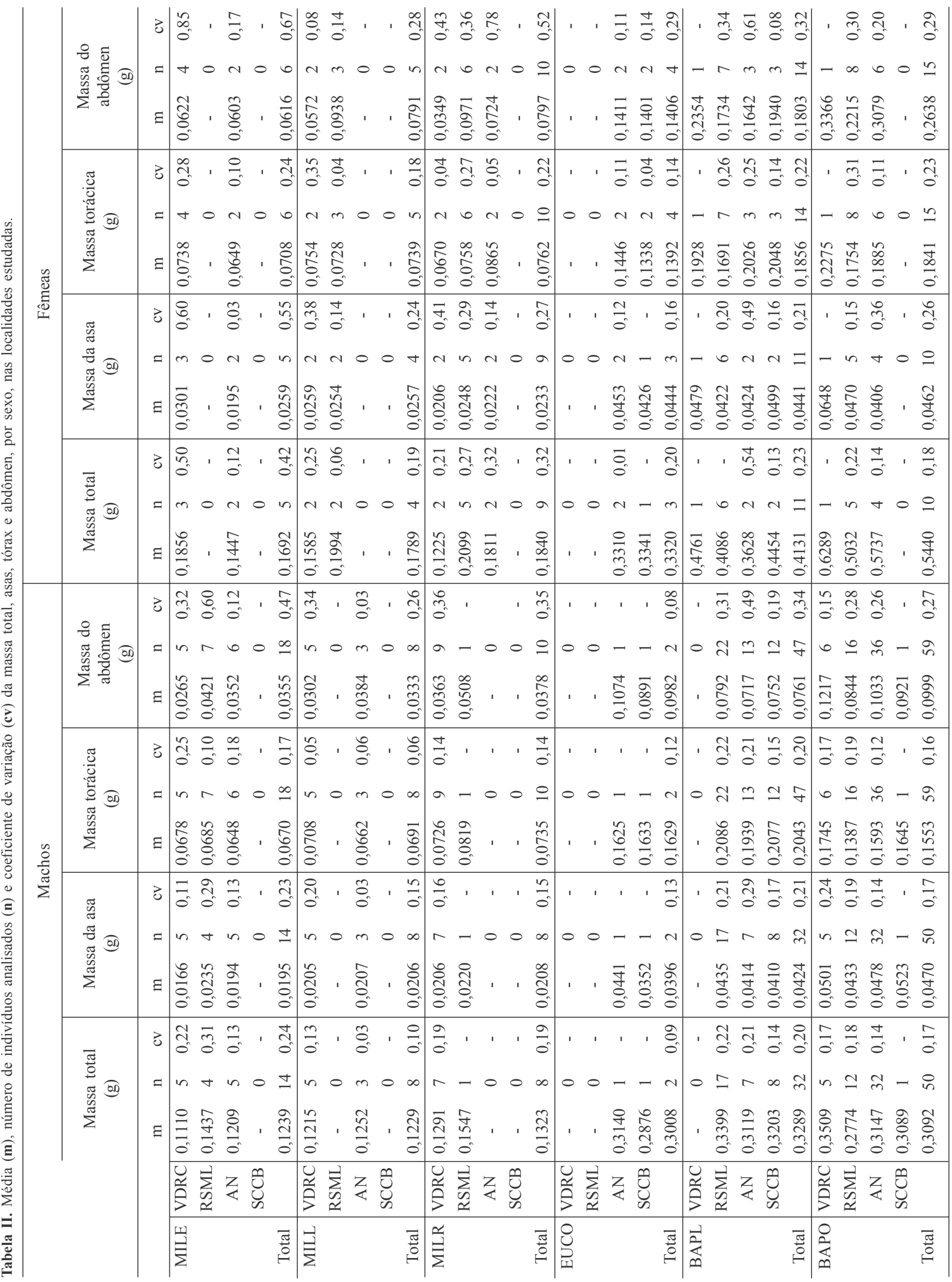




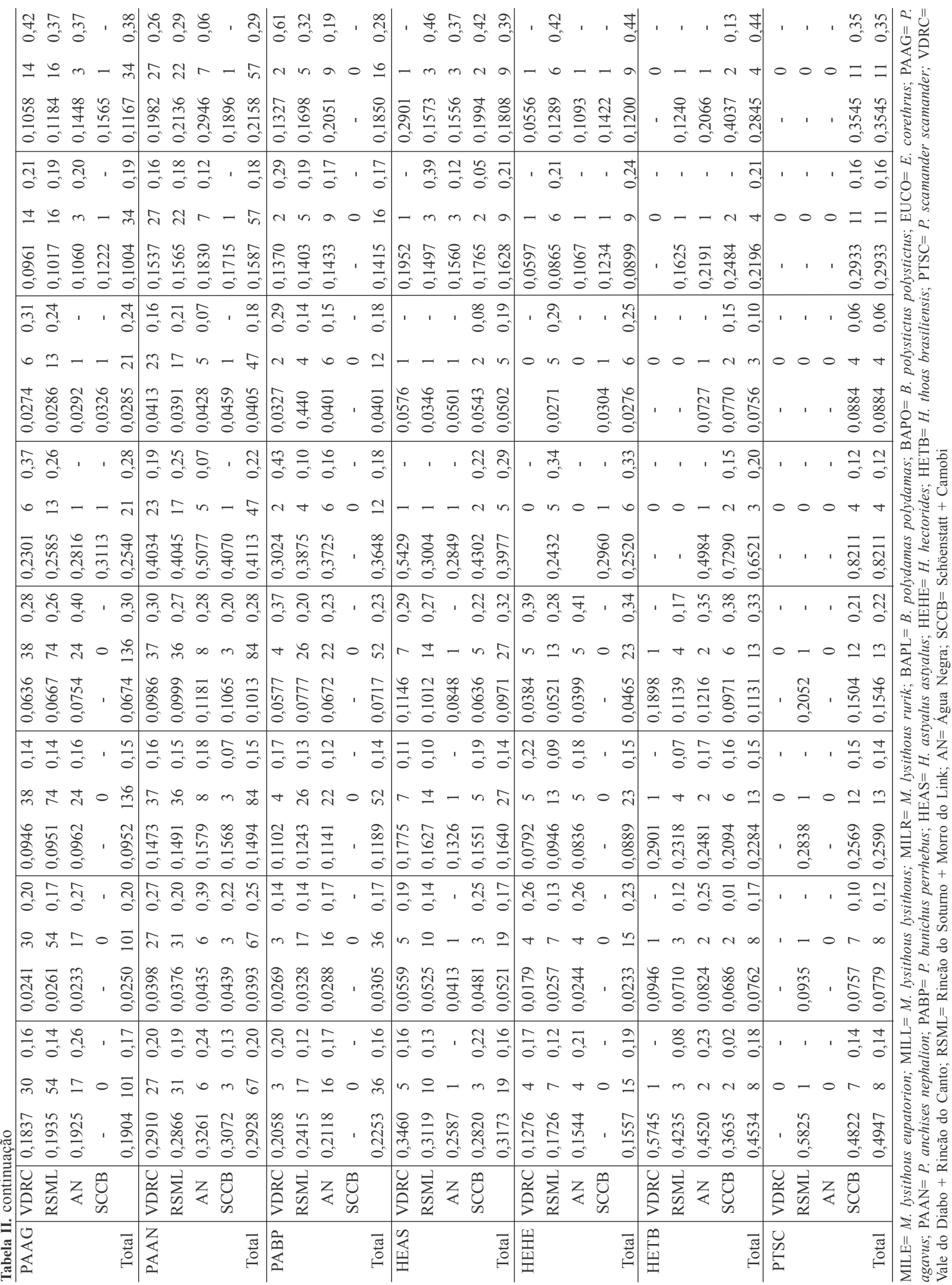


da proporção tórax/abdômen nos machos tornam-se elevados. As diferenças entre SCCB e os demais locais, para os machos, foi estatisticamente significante com ambos os testes. As diferenças entre VDRC, RSML e AN foram significantes somente com o teste LSD. Isso sugere que machos com massas torácicas maiores teriam mais chances de serem encontrados, se estabelecerem ou explorarem áreas menos favoráveis.

Maior massa torácica poderia também significar maior habilidade de vôo, mais chances de acasalar ou fugir de predadores (MARDEN 1989). A fragmentação do hábitat provocada pela diminuição da cobertura vegetal também pode beneficiar machos mais pesados, se a densidade populacional for diminuída. O comportamento para localizar fêmeas, nestes locais, poderá ser alterado com a fragmentação do hábitat. Segundo Dyck \& MATthysen (1999), diferentes tipos de vôo podem ser correlacionados com características morfológicas. Por exemplo, indivíduos que têm comportamento de espera, apresentam tórax relativamente mais pesado do que aqueles que patrulham.

Nas fêmeas, embora a proporção tórax/abdômen diminua com o decréscimo da cobertura e aumento da ação antrópica, ao contrário dos machos, as diferenças entre SCCB e as demais áreas não foram estatisticamente significantes, com ambos os testes. Estudos realizados com Melitaea cinxia (Lepidoptera) mostraram que fêmeas maiores tinham maiores probabilidades de migração (Dyck \& MATthysen 1999). Entretanto, como o vôo dos insetos é claramente multifuncional, comportamentos como forragear, evitar predadores, procurar locais para acasalamento e oviposição, podem ser alvo de pressões seletivas diferentes. Deste modo, pode-se elucidar cenários alternativos para explicar as relações entre a morfologia e a estrutura do hábitat, ou confundir as nossas interpretações para a falta de significância nas fêmeas.

Fica fortalecida a suposição que há uma relação entre a massa das borboletas e o local quando confrontadas as proporções entre a massa das asas com a do tórax (Tabela III C) e do abdômen (Tabela III D). Os aumentos no tamanho do tórax e abdômen foram maiores do que o das asas, à medida que a cobertura vegetal decresce e a antropização aumenta. Isso pode ser observado confrontando-se as quatro comunidades entre si, tanto para os machos como para fêmeas. Esse aumento, na proporção do tórax, em relação à massa das asas, embora tenha sido significante entre SCCB e as demais comunidades, em ambos os sexos, com ambos os testes, fortalece a idéia que uma massa torácica maior poderia ser importante para aumentar o valor adaptativo, ampliando a capacidade de vôo, proporcionando mais chances de acasalar ou de fugir à predação, por exemplo, em ambientes mais antrópicos. Essa projeção qualifica-se mais quando analisamos a proporção da massa das asas em relação ao abdômen (Tabela III D). Nos machos, as diferenças no aumento na proporção do abdômen, analisadas com os dois testes, não foram significativamente maior que o das asas, mantendo-se similar em todos os locais investigados. Entretanto, nas fêmeas SCCB difere significativamente de VDRC e RSML, com ambos os testes.

Segundo Chai \& Srygley (1990), um aumento na musculatura de vôo está associada a um metabolismo com altos custos de energia, que determinam os hábitats ocupados. A relação tórax/abdômen (Tabela III B) das espécies estudadas sugere a ocorrência de pressão seletiva no sentido de maximizar essa disponibilidade de energia, favorecendo indivíduos com maior massa torácica, em qualquer ambiente. Por exemplo, em Papilio machaon e Maculinea arion a redução do tamanho do tórax foi interpretada como uma redução na mobilidade dessas borboletas (DycK \& MATTHYSEN 1999). Além disso, em hábitats fragmentados a mobilidade pode ser favorecida quando as fontes necessárias estão distribuídas em diferentes fragmentos ou partes do ambiente.

Sabe-se que inúmeros fatores da ecologia de insetos dependem diretamente ou indiretamente da habilidade de voar. Seleção na performance de vôo, aparentemente, tem facilitado a radiação adaptativa dos insetos aos variados papéis ecológicos como herbívoros, polinizadores, sugadores de sangue e migrantes (Dudley 2000). Contudo, machos com abdomens proporcionalmente maiores, podem levar desvantagem em ambientes onde o deslocamento rápido, a manobrabilidade do vôo e a busca de parceiros devem ser máximos, e mínimo o gasto de energia. Entretanto, para as fêmeas, embora possa representar uma diminuição na sua sobrevivência, o custo de ter um abdômen maior pode representar uma possibilidade de elevar o seu valor adaptativo. $\mathrm{O}$ custo pode ser compensado por aumentar suas chances de deixar descendentes em ambientes aparentemente menos favoráveis, mas estendendo o seu limite. Deste modo, a mobilidade espacial é essencial para a localização de fontes alimentares espalhadas, tanto para o consumo imediato como para oviposição. Característica nos lepidópteros quando a herbivoria está limitada aos estágios larvais (DudLEy 2000).

Análise de agrupamentos. É importante salientar que $P$. agavus e as subespécies de Mimoides lysithous foram geralmente encontradas em matas ao longo de rios e riachos, ou áreas próximas, não sendo capturadas e nem observadas nas áreas urbanas investigadas (SCCB). Tyler et al. (1994) correlacionam o uso desta especificidade em relação ao hábitat com monitoramento ambiental, associando a sua ausência com a degradação de restingas e/ou matas ao longo dos rios. Algumas espécies podem apresentar atividade intensa de deslocamento dentro das áreas (como P. agavus e P. anchises nephalion) ou de deslocamentos a longa distância (como $H$. astyalus astyalus, $H$. thoas brasiliensis, $P$. scamander scamander, B. polydamas polydamas, $H$. hectorides e $B$. polystictus polystictus). O maior número de capturas de $H$. thoas brasiliensis e P. scamander scamander, em AN e SCCB, pode ser associado como uma adaptação ao aumento da descaracterização dos sistemas florestais. Outras como $P$. bunichus perrhebus podem ter sua presença associada com o aumento de formações abertas, como as observadas em RSML e AN, onde é mais abundante.

A análise dos agrupamentos mostra dois grandes grupos (Fig. 2). Grupo A subdividido em dois subgrupos. Subgrupo $A_{1}$ constituído por duas subdivisões, caracterizadas por tamanhos 
Tabela III. Comparação simultânea dos valores médios (m) obtidos para a massa total (g) (A), para as proporções tórax/abdômen (B), asa/tórax (C) e asa/abdômen (D) através dos testes LSD e Bonferroni (Bonf), para ambos os sexos, nos diferentes locais estudados. Valores médios seguidos pela mesma letra, em cada coluna, não diferem estatisticamente $(\mathrm{p}>0,05)$.

\begin{tabular}{|c|c|c|c|c|c|c|c|c|c|c|c|c|c|c|c|c|c|c|c|c|}
\hline & & & assa & A & & & & & $\operatorname{rax} /$ & $\begin{array}{l}\text { B } \\
\text { Abdômen }\end{array}$ & & & Asa / & $\begin{array}{l}\text { C } \\
\text { Tórax }\end{array}$ & & & & & $\begin{array}{r}D \\
a / A b\end{array}$ & bdômen \\
\hline & & Macl & & & Gême & & & Iach & & Fêmea & & Mach & & & êmea & & & acho & & Fêmeas \\
\hline & $\mathrm{m}$ & $\mathrm{LSD}$ & Bonf & $\mathrm{m}$ & LSD & Bonf & $\mathrm{m}$ & LSD & Bonf & $\mathrm{m} \quad$ LSD & Bonf & $\mathrm{m} \quad \mathrm{LSD}$ & Bonf & $\mathrm{m}$ & LSD & Bonf & $\mathrm{m}$ & LSD & Bonf & $\mathrm{m} \quad$ LSD Bonf \\
\hline VDRC & 0,225 & $\mathrm{a}$ & $\mathrm{a}$ & 0,342 & $\mathrm{a}$ & $\mathrm{a}$ & 1,016 & $\mathrm{a}$ & $\mathrm{a}$ & $0,992 \quad \mathrm{a}$ & $\mathrm{a}$ & $0,967 \quad \mathrm{a}$ & $\mathrm{a}$ & 0,963 & $\mathrm{a}$ & $\mathrm{a}$ & 0,983 & $\mathrm{a}$ & $\mathrm{a}$ & 0,953 a \\
\hline RSML & 0,251 & $\mathrm{~b}$ & $\mathrm{a}$ & 0,340 & $\mathrm{a}$ & a & 1,019 & $\mathrm{~b}$ & $\mathrm{a}$ & 0,989 a,b & $\mathrm{a}$ & $0,963 \quad b$ & $\mathrm{a}$ & 0,963 & $\mathrm{a}$ & $\mathrm{a}$ & 0,981 & $\mathrm{a}$ & a & 0,951 a, \\
\hline AN & 0,255 & $\mathrm{~b}$ & $\mathrm{a}$ & 0,391 & $\mathrm{a}$ & $\mathrm{a}$ & 1,020 & $\mathrm{~b}$ & $\mathrm{a}$ & $0,984 \quad b$ & $\mathrm{a}$ & $0,963 \quad b$ & $\mathrm{a}$ & 0,959 & $\mathrm{a}$ & $\mathrm{a}$ & 0,982 & $\mathrm{a}$ & $\mathrm{a}$ & $0,944 a, b \quad a, b$ \\
\hline SCCB & 0,361 & $\mathrm{c}$ & $\mathrm{b}$ & 0,560 & $\mathrm{~b}$ & b & 1,039 & $\mathrm{c}$ & b & $0,987 \mathrm{a}, \mathrm{b}$ & $\mathrm{a}$ & $0,947 \quad \mathrm{c}$ & b & 0,945 & $\mathrm{~b}$ & b & 0,981 & $\mathrm{a}$ & $\mathrm{a}$ & $0,930 \quad b$ \\
\hline
\end{tabular}

$\mathrm{VDRC}=$ Vale do Diabo + Rincão do Canto; RSML= Rincão do Soturno + Morro do Link; AN= Água Negra; SCCB= Schöenstatt +

e proporções similares, bem como padrões miméticos de coloração também semelhantes e ocupando ambientes cuja principal característica é a umidade alta, principalmente para $P$. agavus. Uma é formada pelas subespécies de Mimoides lysithous que habitam preferencialmente florestas naturais pouco perturbadas como mata tropical temperada, hábitats ribeirinhos ou próximos de rios ou riachos. Voam acima da vegetação, com muitos deslizamentos (vôo planado). O vôo é retilíneo, rápido e trêmulo. Movimento à longa distância é desconhecido. O macho persegue e subjuga fisicamente a fêmea durante os cortejamentos. A outra é constituída por $H$. hectorides que preferencialmente habita mata sazonal densa $\mathrm{e}$ pouco perturbada, com deslocamentos longos, e P. agavus, limitada a hábitats úmidos e sombreados. As duas espécies voam no interior da vegetação sem deslizamentos. O vôo é normalmente vagaroso, errático, com as asas para cima. Machos ficam pousados aguardando as fêmeas, com perseguição aérea e subjugação delas (BROwN Jr. 1992; TYLER et al. 1994).

O subgrupo $\mathrm{A}_{2}$ é formado pelas espécies $P$. bunichus perrhebus, E. corethrus, B. polydamas polydamas, $P$. anchises nephalion e $H$. astyalus astyalus que são, geralmente, encontradas em ambientes antrópicos como bosques com pouca umidade, locais com vegetação perturbada, formações abertas, parques urbanos e jardins. Neste subgrupo os machos ficam pousados aguardando as fêmeas, com perseguição aérea e utilizando persuasão química para acalmá-las (exceto os machos de E. corethrus que perseguem e subjugam as fêmeas) (Brown Jr. 1992; Tyler et al. 1994). Esse subgrupo também apresenta duas divisões. A primeira é formada por B. polydamas polydamas, $H$. astyalus astyalus, $P$. anchises nephalion e $P$. bunichus perrhebus. Parides bunichus perrhebus e P. anchises nephalion, normalmente, foram encontradas no interior ou borda de mata, voando sem deslizamentos e sem deslocamentos longos. $\mathrm{O}$ vôo normalmente é retilíneo, rápido e trêmulo, mas também pode ser vagaroso, errático, com as asas para cima. $B$. polydamas polydamas e $H$. astyalus astyalus apresentam vôo retilíneo, rápido e trêmulo, normalmente, acima da vegetação, com deslizamentos e deslocamentos longos ou aos saltos. A segunda é constituída exclusivamente por E. corethrus, que se sobressai das demais por ser encontrada preferencialmente em campos e prados (BRown Jr. 1992; TYler et al. 1994).

As espécies do grupo $\mathrm{B}$, mais heterogêneo, apresentam uma característica bastante particular, ou seja, a de ocuparem ambientes cuja ação antrópica é suficientemente grande para descaracterizar os ambientes naturais originais. Ocupa fundamentalmente ambientes antrópicos como bosques com pouca umidade, locais com vegetação bastante perturbada, formações abertas, parques urbanos e jardins. Voam normalmente acima da vegetação com deslizamentos e deslocamentos longos. O vôo é rápido e aos saltos. Os machos ficam pousados aguardando as fêmeas, com perseguição aérea da fêmea, utilizam persuasão química para acalmá-las (H. thoas brasiliensis), ou o macho persegue e subjuga fisicamente a fêmea (P. scamander scamander, B. polystictus polystictus) (BROWN Jr. 1992; TyLER et al. 1994).

Como consideração final podemos inferir que o subgrupo $\mathrm{A}_{1}$ é distinguido principalmente pelo tipo de corte que os machos realizam, enquanto $A_{2}$ tem como característica principal a de se adaptar a ambientes com vegetação perturbada, evidentemente não tão perturbada como as ocupadas pelo grupo B.

\section{CONCLUSÕES}

Embora o tamanho corporal tenha sido bastante estudado em borboletas, ainda existem exemplos marcantes como Papilioninae que podem fornecer informações novas.

Este trabalho associa quantitativamente a massa dos indivíduos e suas partes ao sexo, aos locais de captura e ao comportamento. Tanto as asas como o tórax e abdômen contribuem para estabelecer diferenças observadas entre as espécies e entre os sexos. As fêmeas apresentam maior tamanho corporal que os machos. A maior variação, nas fêmeas, está associada ao tamanho do abdômen (dando-lhes um aspecto mais robusto), enquanto que nos machos, está no tamanho do tórax. A diferença no tamanho e na forma do corpo afeta diferentemente a adaptação das borboletas. E ainda sugere 
que o tamanho corporal das fêmeas está dirigido para a reprodução, enquanto nos machos para uma melhor performance de vôo. Em ambos os sexos, a proporção do tórax é significativamente influenciada pelo hábitat. Conforme os hábitats se tornam mais antrópicos e com menor cobertura vegetal, a proporção aumenta. Nas fêmeas a proporção de aumento do abdômen é influenciada pelo tipo de hábitat, ao contrário dos machos que é similar em todos os locais investigados.

Os agrupamentos formados a partir da proporção torácica e abdominal reúnem espécies em dois grandes agrupamentos com tamanhos e proporções similares. Um, que pode ser associado a ambientes de floresta, cuja principal característica é a umidade alta. Outro, mais heterogêneo, que apresenta uma característica bastante particular, cujos representantes ocupam ambientes onde a ação antrópica é suficientemente grande para descaracterizar a vegetação natural original.

Todavia, o estudo também mostra que são necessárias investigações que agreguem mecanismos pelos quais essas características são selecionadas. Igualmente, faz-se necessário um estudo detalhado sobre a variabilidade genética, que determina os elementos da distribuição espacial, e a fragmentação das populações naturais, que influencia as variações no tamanho.

Agradecimentos. Agradecimentos aos doutores Alexandre Specht (Universdade de Caxias do Sul, RS) e Carla M. Penz (Dep. of Invertebrate Zoology, Milwaukee Public Museum) e ao colega José A. Teston pelas críticas e sugestões efetuadas. Este trabalho teve suporte financeiro da CAPES/PIDCT.

\section{REFERÊNCIAS}

Andersen, N. M. 1994. The evolution of sexual size dimorphism and mating systems in water striders (Hemiptera, Gerridae): a phylogenetic approach. Ecoscience 1: 208-214.

AraK, A. 1994. Sexual dimorphism in body size: a model and a test. Evolution 42: 820-825.

ArCher, J. 1988. The behavioral biology of aggression. Cambridge, Cambridge University Press, $257 \mathrm{p}$.

Barbault, R. 1988. Body size, ecological constraints, and the evolution of life-history strategies, p. 261-286. In: M. K. Hecht; B. Wallace, \& G. T. Prance, (eds.). Evolutionary Biology. New York, Plenum.

Brown Jr., K. S. 1992. Borboletas da Serra do Japi: diversidade, hábitats, recursos alimentares e variação temporal, p. 142-186. In: L. P. C. Morellato (org.). Historia natural da Serra do Japi: ecologia e preservação de uma área florestal no sudoeste do Brasil. Campinas, Editora da UNICAMP/FAPESP.

Calder, W. A. 1984. Size, Function and Life History. Cambridge, Harvard University Press, $431 \mathrm{p}$

Chai, P. \& R. B. SRygley. 1990. Predation and the flight, morphology, and temperature of Neotropical rain forest butterflies. American Naturalist 135: 748-765.

Convey, P. 1989. Influences on the choice between territorial and satellite behaviour in male Libellula quadrimaculata Linn. (Odonata: Libellulidae). Behaviour 109: 125-141.

Cronin, H. 1995. A formiga e o pavão - altruísmo e seleção sexual de Darwin até hoje. Campinas, Papirus, 643 p.

DARWIN, C. 1871. The descent of man, and selection in relation to sex. Princeton, Princeton University Press, 405 p.

Dudley, R. 2000. The biomechanics of insect flight. Princeton, Princeton University Press, $476 \mathrm{p}$.

Dyck, H. V. \& E. Matthysen. 1999. Habitat fragmentation and insect flight: a changing "design" in a changing landscape? TREE 14(5): 172-174.

Eldredge, N. 1986. Information, economics, and evolution. Annual Review of Ecology \& Systematics 17: 351-369.

Fairbairn, D. J. 1990. Factors influencing sexual size dimorphism in temperate water striders. American Naturalist 136: 61-86.

Fairbairn, D. J. 1992. The origins of allometry: size and shape polymorphism in the common waterstrider, Gerris remigis Say (Heteroptera, Gerridae). Biological Journal of the Linnean Society 45: 167-168.

Feeny, P. 1991. Chemical constraints on the evolution of Swallowtail butterflies, p. 315-340. In: P. W. Price; T. M. Lewinsohn; G. W. Fernandes, \& W. W. Benson (eds.). Plant-animal interactions: evolutionary ecology in tropical and temperate regions. New York, John Wiley \& Sons.

Funk, V. A. \& D. R. Brooks. 1990. Phylogenetic systematics as the basis of comparative biology. Washington, Smithsonian Institution Press, $45 \mathrm{p}$.

Gadgil, M. \& Bossert, W. H. 1970. Life historical consequences of natural selection. American Naturalist 104: 1-24.

Ghiselin, M. T. 1974. The economy of nature and evolution of sex. Berkeley, University of California Press, $346 \mathrm{p}$.

Gould, S. J. 1966. Allometry and size in ontogeny and phylogeny. Biological Review 41: 587-640.

Hanken, J. \& D. B. WaKe. 1993. Miniaturization of body size: organismal consequences and evolutionary significance. Annual of Review Ecology and Systematics 24: 501-519.

Hendrick, A. V. \& E. J. Temeles. 1989. The evolution of sexual dimorphism in animals: hypotheses and tests. TREE 4: 136-138.

Huey, R. B. \& E. R Pianka. 1981. Ecological consequences of foraging mode. Ecology 62: 991-999.

KLEIN, R. M. 1984. Aspectos dinâmicos da vegetação do sul do Brasil. Sellowia 36: 5-54.

La Barberra, M. 1989. Analyzing body size as a factor in ecology and evolution. Annual Review of Ecology and Systematics 88: 92 99.

LANDE, R. 1979. Quantitative genetic analysis of multivariate evolution applied to brain: body size allometry. American Naturalist 33: 402-416.

Lederhouse, R. C. 1982. Territorial defense and behavior of the black swallowtail butterfly, Papilio polyxenes. Behavioral Ecology and Sociobiology 10: 109-118.

Marden, J. H. 1989. Effects of load-lifting constraints on the mating system of a dance fly. Ecology 70: 496-502.

Nijhout, H. F. \& J. Emlen. 1998. Competition among body parts in the development and evolution of insect morphology. Proceedings of the National Academy of Sciences USA 35: 3685-3689.

Peters, R. H. 1983. The ecological implications of body size. Cambridge, Cambridge University Press, 329 p.

Reeve, J. P. \& D. J. Fairbairn. 1996. Sexual size dimorphism as a correlated response to selection on body size: an empirical test of the quantitative genetic model. Evolution 50(5): 1927-1938.

Reiss, M. J. 1989. The allometry of growth and reproduction. Cambridge, Cambridge University Press, $182 \mathrm{p}$.

Riska, B. 1989. Composite traits, selection response, and evolution. Evolution 43: 1172-1191.

Schmidt-Nielsen, K. 1984. Scaling: why is animal size so important? Cambridge, Cambridge University Press, $241 \mathrm{p}$.

Schoener, T. W. 1969. Models of optimum size for solitary predators. American Naturalist 103: 227-313.

Schwartz, G. \&. R. A. Di Mare. 2001. Diversidade de quinze espécies de borboletas (Lepidoptera, Papilionidae) em sete comunidades de Santa Maria, RS. Ciência Rural 31(1): 49-55.

Scriber, J. M.; R. C. Lederhouse \& R. H. Hagen. 1991. Foodplants and Evolution within Papilio glaucus and Papilio troilus groups (Lepidoptera: Papilionidae), p. 341-73. In: P. W. Price; T. M. 
Lewinsohn; G. W. Fernandes \& W. W. Benson, (eds.). Plant-animal interactions: evolutionary ecology in tropical and temperate regions. New York, John Wiley \& Sons.

Selander, R. K. 1972. Sexual selection and dimorphism in birds, p. 180230. In: B. G. Campbell (org.). Sexual selection and the descent of man 1871-1971. London, Heinemann.

Sneath, P. H. A. \& R. R. Sokal, 1973. Numerical taxonomy. San Francisco, W. H. Freeman \& Co, 573 p.

Srygley, R. B. \& P. Chai. 1990. Flight morphology of Neotropical butterflies: palatability and distribution of mass to the thorax and abdomen. Oecologia 84: 491-499.

SRygley, R. B. \& R. Dudley. 1993. Correlations of the position of center of body mass with butterfly escape tatics. Journal of Experimental of Biology 174: 155-166.

Stearns, S. C. \& R. E. Crandall. 1984. Plasticity of age and size at sexual maturity: a life-history response to unavoid stress, p. 13-33. In: G. W. Pоттs \& R. J. Wооттоn (eds.). Fish Reproduction: strategies and tactics. New York, New York Academic Press.

Teston, J. A. \& E. Corseuil. 1998. Lista documentada dos papilionídeos (Lepidoptera, Papilionidae) do Rio Grande do Sul, Brasil. Biociências
6: 81-94.

Tyler, H. A.; K. S. Brown Jr. \& K. H. Wilson. 1994. Swallowtail butterflies of the Americas: a study in biological dynamics, ecological diversity, biosystematics and conservation. Gainesville, Scientific Publishers, 376 p.

Valentin, J. L. 2000. Ecologia numérica: uma introdução à análise multivariada de dados ecologicos. Rio de Janeiro, Interciência, $117 \mathrm{p}$.

VitT, L. J. 1983. Tail loss in lizards: the significance of foraging and predator escape modes. Herpetologica, 39: 151-162.

VitT, L. J. \& J. D. Congdon. 1978. Body shape, reproductive effort and relative clutch mass in lizards: resolution of a paradox. American Naturalist 117: 595-608.

Webi, P. W. 1984. Body form, locomotion and foraging in aquatic vertebrates. American Zoologist 24: 107-120.

ZAR, J. H. 1999. Biostatistical analysis. New Jersey, Prentice-Hall. $663 \mathrm{p}$.

ZENG, Z. 1988. Long-term correlated responses, interpopulation covariation, and interspecific allometry. Evolution 42: 363-374.

Recebido em 10.II.2004; aceito em 15.IX.2004 